\title{
A finite action for three dimensional gravity with a minimally coupled scalar field
}

\author{
Jack Gegenberg ${ }^{\S}$, Cristián Martínez ${ }^{\ddagger}$, and Ricardo Troncoso ${ }^{\ddagger}$ \\ $\S$ Department of Mathematics and Statistics and Department of Physics, \\ University of New Brunswick, Fredericton, NB Canada E3B 5 A3 \\ ${ }_{\ddagger}$ Centro de Estudios Cientificos (CECS), Casilla 1469, Valdivia, Chile \\ e-mail: lenin@math.unb.ca, martinez@cecs.cl, ratron@cecs.cl
}

\begin{abstract}
Three-dimensional gravity with a minimally coupled self-interacting scalar is considered. The fall-off of the fields at infinity is assumed to be slower than that of a localized distribution of matter, so that the asymptotic symmetry group is the conformal group.

The counterterm Lagrangian needed to render the action finite is found by demanding that the action attain an extremum for the boundary conditions implied by the above fall-off of the fields at infinity. These counterterms explicitly depend on the scalar field. As a consequence, the Brown-York stress-energy tensor acquires a non trivial contribution from the matter sector.

Static circularly symmetric solutions with a regular scalar field are explored for a one-parameter family of potentials. Their masses are computed via the Brown-York quasilocal stress-energy tensor, and they coincide with the values obtained from the Hamiltonian approach. The thermal behavior, including the transition between different configurations, is analyzed, and it is found that the scalar black hole can decay into the BTZ solution irrespective of the horizon radius.

It is also shown that the AdS/CFT correspondence yields the same central charge as for pure gravity.
\end{abstract}




\section{Introduction}

The asymptotic behavior of gravity with a negative cosmological constant has been extensively explored since the 1980's, initially in the context of symmetries and conserved charges [1]-16, and now in relation with the AdS/CFT correspondence [17, 18, 19. The usual assumption is that matter fields fall off sufficiently fast to insure that conserved charges can be written as surface integrals involving only the metric and its derivatives. Here we deal with a case where the matter fields drop off so slowly in the asymptotic region, that they add a nontrivial contribution to the conserved charges, as well as to the Euclidean action. This issue is addressed for three-dimensional gravity with a minimally coupled self-interacting real scalar field. This theory admits interesting asymptotically AdS solutions including black holes [20] and a sort of degenerate ground state, both with non-trivial regular scalar fields. To distinguish those black holes with a non-trivial scalar field from BTZ black holes (which are solutions with the scalar field constant) we call the former 'scalar black holes'. With the exception of the BTZ geometries, the solutions have a slower than expected fall-off to AdS in the asymptotic region. The scalar potential is constructed so that this weaker fall-off is preserved by the action of the Virasoro group generated by the asymptotic Killing vector fields.

Since the scalar field behaves as $O\left(r^{-1 / 2}\right)$, it necessarily contributes to the action and its variations in the asymptotic region, and the counterterms of [8] will not yield a finite action or charges [9] in this case. In the next section, we obtain suitable counterterms, which depend explicitly on the scalar field, from the requirement that the action must be functionally differentiable for both metric and scalar fields which obey these weaker fall-off conditions. This means that the quasilocal stress-energy tensor defined in Ref. 21] also acquires a contribution coming from the matter field. In Section [3, we will first briefly review the asymptotic conditions of Ref. [20], and we will display static circularly symmetric solutions with a regular scalar field for a oneparameter family of potentials, and compute the mass using the Brown-York stress-energy tensor. In Section 4, we will calculate the action for the Wickrotated solutions, and use this to discuss some aspects of the thermodynamics

of the solutions. It turns out that there is a nonvanishing probability for the decay of a scalar black hole into the BTZ black hole. The central charge is computed via the AdS/CFT correspondence, yielding the same value as one would obtain for pure gravity [4]. 


\section{Action, counterterms and quasilocal stress- energy tensor}

\subsection{Asymptotic fall-off conditions}

The asymptotic behavior of three-dimensional pure gravity with a negative cosmological constant is described by the Brown-Henneaux boundary conditions [4, which are left invariant under a symmetry group generated by the following asymptotic Killing vectors

$$
\begin{aligned}
\eta^{t} & =l\left[T^{+}+T^{-}+\frac{l^{2}}{2 r^{2}}\left(\partial_{+}^{2} T^{+}+\partial_{-}^{2} T^{-}\right)\right]+O\left(r^{-4}\right) \\
\eta^{r} & =-r\left(\partial_{+} T^{+}+\partial_{-} T^{-}\right)+O\left(r^{-1}\right) \\
\eta^{\varphi} & =T^{+}-T^{-}-\frac{l^{2}}{2 r^{2}}\left(\partial_{+}^{2} T^{+}-\partial_{-}^{2} T^{-}\right)+O\left(r^{-4}\right)
\end{aligned}
$$

where $T^{+}\left(x^{+}\right)$and $T^{-}\left(x^{-}\right)$generate two independent copies of the Virasoro algebra and $x^{ \pm}=t / l \pm \varphi$.

These conditions hold also for localized matter fields which fall-off sufficiently fast at infinity, so as to give no contributions to the surface integrals defining the generators of the asymptotic symmetries. With these assumptions, the charges that generate the asymptotic symmetries involve only the metric and its derivatives, and their algebra corresponds to be a central extension of the asymptotic symmetry algebra, where the central charge is given by

$$
c=\frac{3 l}{2 G} \text {. }
$$

However, there are instances in which the matter fields modify the asymptotic behavior of the metric. A well-known example is the electrically charged black hole, where the metric has a logarithmic divergence [22, 23, 24]. In those cases, there is a possibility of having divergent contributions coming both from the gravitational and matter actions. In these situations, the asymptotic conditions must be such that the sum of both contributions converges.

The case in which the fall-off of the fields at infinity is slower than that of a localized distribution of matter was analyzed in Ref. [20]. The matter sector was assumed to be given by a single self-interacting scalar field minimally 
coupled to three dimensional gravity, with the action

$$
I_{B u l k}[g, \phi]=\frac{1}{\pi G} \int d^{3} x \sqrt{-g}\left[\frac{R}{16}-\frac{1}{2}(\nabla \phi)^{2}-V(\phi)\right] .
$$

Black hole solutions with a nontrivial scalar field were found for a oneparameter family of potentials $V(\phi)$, whose asymptotic behavior belongs to the following class

$$
\begin{array}{cl}
\quad \phi=\frac{\chi}{r^{1 / 2}}-\alpha \frac{\chi^{3}}{r^{3 / 2}}+O\left(r^{-5 / 2}\right) & \\
g_{r r}=\frac{l^{2}}{r^{2}}-\frac{4 l^{2} \chi^{2}}{r^{3}}+O\left(r^{-4}\right) & g_{t t}=-\frac{r^{2}}{l^{2}}+O(1) \\
g_{t r}=O\left(r^{-2}\right) & g_{\varphi \varphi}=r^{2}+O(1) \\
g_{\varphi r}=O\left(r^{-2}\right) & g_{t \varphi}=O(1)
\end{array}
$$

where $\chi=\chi(t, \varphi)$, and $\alpha$ is an arbitrary constant. Note that the asymptotic behavior of $g_{r M}$ has a slower fall-off than that discussed by Brown and Henneaux ${ }^{1}$. Consistency of these relaxed asymptotic boundary conditions with the field equations is sufficient to fix the potential $V(\phi)$ to be of the form

$$
V(\phi)=-\frac{1}{8 l^{2}}-\frac{3}{8 l^{2}} \phi^{2}-\frac{1}{2 l^{2}} \phi^{4}+\phi^{6} U(\phi),
$$

where $U\left(\phi^{2}\right)$ could be any function which is smooth in a neighborhood of $\phi=0$. In spite of the fact that $V(\phi)$ could even be unbounded from below, this potential satisfies the conditions that guarantee the perturbative stability of AdS space [25], 26].

Remarkably, it was found that this set of conditions is also left invariant under the Virasoro algebra generated by the asymptotic Killing vectors (11). Furthermore, using the Regge-Teitelboim approach [27, it was found that the generators of the asymptotic symmetries acquire a contribution from the scalar field ${ }^{2}$

$$
\begin{aligned}
Q(\xi)= & \frac{1}{16 \pi G} \int d \varphi\left\{\frac{\xi^{\perp}}{l r}\left(\left(g_{\varphi \varphi}-r^{2}\right)-2 r^{2}\left(l g^{-1 / 2}-1\right)\right)+2 \xi^{\varphi} \pi_{\varphi}^{r}\right. \\
& \left.+\xi^{\perp} \frac{2 r}{l}\left[\phi^{2}-2 l \frac{\phi \partial_{r} \phi}{\sqrt{g_{r r}}}\right]\right\}
\end{aligned}
$$

\footnotetext{
${ }^{1}$ Henceforth, capital latin indices stand for three-dimensional spacetimes coordinates, and greek indices label the coordinates at the boundary.

${ }^{2}$ Eq.(7) is a slightly improved version of the expression found in Ref. [20, because it does not depend on the parameter $\alpha$ appearing in Eq.(4).
} 
and the algebra of these canonical generators have the standard central extension given by Eq. (2)

In the next subsection, we use the background independent method of 8 to find the counterterm Lagrangian needed to render the action finite (see also [9]). In contrast to the case of a localized distribution of matter, it is shown that the correction terms acquire contributions depending explicitly on the scalar field. This allows us to construct an alternative to Eq. (17) which we obtain from the Brown-York stress-energy tensor.

\subsection{Counterterm action}

In analogy with the counterterm prescription in Refs. 8, 9, we consider the following action

$I=\frac{1}{\pi G} \int_{M} d^{3} x \sqrt{-g}\left(\frac{R}{16}-\frac{1}{2}(\nabla \phi)^{2}-V(\phi)\right)+\frac{1}{8 \pi G} \int_{\partial M} d^{2} x \sqrt{-\gamma} \Theta+I_{c t}[\gamma, \phi]$,

where the boundary term containing the trace of the extrinsic curvature $\Theta$ is required to fix Dirichlet conditions for the metric. Owing to the asymptotic behavior of the fields, the counterterm action $I_{c t}$ is assumed to depend not only on the boundary metric $\gamma_{\mu \nu}$, but also on the scalar field.

The strategy for obtaining the counterterm action will be to require that the action (88) should have an extremum for the solutions satisfying the asymptotic conditions (4) and (5D). It turns out that this approach also ensures the convergence of the action.

The variation of (8) is given by

$$
\delta I=\int_{M} E_{I} \delta \Phi^{I}+\int_{\partial M} d^{2} x \pi^{\mu \nu} \delta \gamma_{\mu \nu}-\frac{1}{\pi G} \int_{\partial M} d^{2} x \sqrt{-\gamma} \hat{n}^{M} \partial_{M} \phi \delta \phi+\delta I_{c t},
$$

where $\Phi^{I}:=\left\{g_{M N}, \phi\right\}$ are the dynamical fields and $E_{I}$ are the corresponding equations of motion. The spacetime metric $g_{M N}$ has been decomposed in a radial ADM foliation as

$$
d s^{2}=N^{2} d r^{2}+\gamma_{\mu \nu}\left(d x^{\mu}+N^{\mu} d r\right)\left(d x^{\nu}+N^{\nu} d r\right),
$$

and the boundary momenta are

$$
\pi^{\mu \nu}:=\frac{1}{16 \pi G} \sqrt{-\gamma}\left(\Theta \gamma^{\mu \nu}-\Theta^{\mu \nu}\right) .
$$


Here the extrinsic curvature $\Theta^{\mu \nu}$, defined through the covariant derivative of the outward pointing normal vector $\hat{n}_{M}=\left(0,1 / \sqrt{g^{r r}}, 0\right)$ to the boundary $\partial M$, is

$$
\Theta^{\mu \nu}:=\frac{1}{2}\left(\nabla^{\mu} \hat{n}^{\nu}+\nabla^{\nu} \hat{n}^{\mu}\right)
$$

Hence the action attains an extremum provided the variation of the counterterm action satisfies

$$
\delta I_{c t}=-\int_{\partial M} d^{2} x \pi^{\mu \nu} \delta \gamma_{\mu \nu}+\frac{1}{\pi G} \int_{\partial M} d^{2} x \sqrt{-\gamma} \hat{n}^{M} \partial_{M} \phi \delta \phi,
$$

which, by virtue of the asymptotic conditions (4) and (15), becomes

$\delta I_{c t}=-\frac{1}{16 \pi G} \int_{\partial M} d^{2} x\left(\frac{1}{l^{2}} \delta h_{\varphi \varphi}-\delta h_{t t}\right)-\frac{1}{4 \pi G l^{2}} \int_{\partial M} d^{2} x\left(r \delta \chi^{2}+(1+3 \alpha) \delta \chi^{4}\right)$.

Here $h_{\mu \nu}(t, \varphi)$ denotes the deviation from the AdS asymptotic metric. The first term at the right hand side of (13) corresponds to the variation of the volume of $\partial M$

$$
\delta\left[\int_{\partial M} d^{2} x \sqrt{-\gamma}\right]=\frac{l}{2} \int_{\partial M} d^{2} x\left(\frac{1}{l^{2}} \delta h_{\varphi \varphi}-\delta h_{t t}\right),
$$

and using the asymptotic form of the scalar field, it is simple to see that the second term in (13) corresponds to the variation of a covariant expression that reads

$$
\frac{1}{8 \pi G l} \int_{\partial M} d^{2} x \sqrt{-\gamma}\left(2 l \phi \hat{\eta}^{M} \partial_{M} \phi-\phi^{2}\right),
$$

which depends on the boundary metric as well as on the scalar field.

Therefore, the counterterm action can be written as

$$
\begin{aligned}
I_{c t} & =I_{c t}^{G}+I_{c t}^{\phi} \\
& =\frac{1}{8 \pi G l}\left[-\int_{\partial M} d^{2} x \sqrt{-\gamma}+\int_{\partial M} d^{2} x \sqrt{-\gamma}\left(2 l \phi \hat{\eta}^{M} \partial_{M} \phi-\phi^{2}\right)\right],
\end{aligned}
$$

where $I_{c t}^{G}$ is the counterterm required for asymptotically AdS spacetimes in the sense of Brown and Henneaux, and $I_{c t}^{\phi}$ is required to cancel the variation coming from the kinetic term in the bulk, provided the relaxed asymptotic conditions (4) and (5) are imposed.

The presence of $I_{c t}^{\phi}$ implies that the surface integrals defining the conserved charges acquire a nontrivial contribution coming from the matter sector. This will be explicitly demonstrated in the next subsection, where we employ the Brown-York approach. 


\subsection{Quasilocal stress-energy tensor}

The quasilocal stress-energy tensor $T^{\mu \nu}$ associated with a region of the spacetime that is bounded by a surface with metric $\gamma_{\mu \nu}$ is given by

$$
T^{\mu \nu}=\frac{2}{\sqrt{-\gamma}} \frac{\delta I}{\delta \gamma_{\mu \nu}}
$$

Considering a radial ADM foliation as in Eq. (10), the variation of the action on-shell is

$$
\frac{\delta I}{\delta \gamma_{\mu \nu}}=\int_{\partial M} d^{2} x \pi^{\mu \nu}+\frac{\delta I_{c t}}{\delta \gamma_{\mu \nu}}
$$

where $\pi^{\mu \nu}$ is given by (11). Hence, using Eq. (11), the stress-energy tensor reads:

$$
T^{\mu \nu}=\frac{1}{8 \pi G}\left(\Theta \gamma^{\mu \nu}-\Theta^{\mu \nu}\right)+\frac{2}{\sqrt{-\gamma}} \frac{\delta I_{c t}}{\delta \gamma_{\mu \nu}},
$$

which by virtue of $I_{c t}$ in Eq. (14), can be written as

$$
\begin{aligned}
T^{\mu \nu} & =T_{G}^{\mu \nu}+T_{\phi}^{\mu \nu} \\
& =\frac{1}{8 \pi G}\left(\Theta \gamma^{\mu \nu}-\Theta^{\mu \nu}-\frac{1}{l} \gamma^{\mu \nu}\right)+\frac{1}{8 \pi G l} \gamma^{\mu \nu}\left(2 l \phi \hat{\eta}^{M} \partial_{M} \phi-\phi^{2}\right)
\end{aligned}
$$

The conserved charges can be constructed by choosing an ADM foliation at $\partial M$ with spacelike surfaces $\Sigma$, so that

$$
\gamma_{\mu \nu} d x^{\mu} d x^{\nu}=-N_{\Sigma}^{2} d t^{2}+\sigma\left(d \varphi+N_{\Sigma}^{\varphi} d t\right)^{2} .
$$

Hence

$$
Q_{B Y}(\xi)=\int_{\Sigma} d x \sqrt{\sigma} u^{\mu} \xi^{\nu} T_{\mu \nu}
$$

Here $u^{\mu}$ is the timelike unit normal to $\Sigma$, and $\xi^{\mu}$ is a Killing vector of the boundary. Thus, choosing $\xi^{\mu}=N_{\Sigma} u^{\mu}$, the mass is written as a surface integral,

$$
\begin{aligned}
M & =\int_{\Sigma} d x \sqrt{\sigma} N_{\Sigma} u^{\mu} u^{\nu}\left(T_{\mu \nu}^{G}+T_{\mu \nu}^{\phi}\right) \\
& =M_{G}+M_{\phi} .
\end{aligned}
$$

Note that the mass acquires a nontrivial contribution from the matter sector.

In what follows, the previous formalism is tested for some exact solutions possessing the asymptotic behavior given by (4) and (5) for a one-parameter family of potentials of the form (6) . 


\section{Testing the counterterms with exact solu- tions}

Exact solutions for which the metric and the scalar field satisfy the asymptotic conditions (44) and (5) can be obtained for a particular one-parameter family of potentials of the form [20]

$$
V_{\nu}(\phi)=-\frac{1}{8 l^{2}}\left(\cosh ^{6} \phi+\nu \sinh ^{6} \phi\right) .
$$

This potential belongs to the class (6), and different forms of $U\left(\phi^{2}\right)$ are obtained for different values of the dimensionless parameter $\nu$. This parameter can be interpreted as the self-interacting coupling constant in the conformal frame [20].

\subsection{Black hole with a regular non-constant scalar field}

For the range $\nu>-1$, the potential is unbounded from below and satisfies the conditions that guarantees the perturbative stability of AdS space [26]. In this case, a static circularly symmetric black hole solution, dressed with a scalar field which is regular everywhere ${ }^{3}$ was found in Ref. 20. The scalar field is given by

$$
\phi=\operatorname{arctanh} \sqrt{\frac{B}{H(r)+B}},
$$

where $B$ is a nonnegative integration constant and

$$
H(r)=\frac{1}{2}\left(r+\sqrt{r^{2}+4 B r}\right) .
$$

The metric reads

$$
d s^{2}=-\left(\frac{H}{H+B}\right)^{2} F(r) d t^{2}+\left(\frac{H+B}{H+2 B}\right)^{2} \frac{d r^{2}}{F(r)}+r^{2} d \varphi^{2}
$$

with

$$
F=\frac{H^{2}}{l^{2}}-(1+\nu)\left(\frac{3 B^{2}}{l^{2}}+\frac{2 B^{3}}{l^{2} H}\right) .
$$

\footnotetext{
${ }^{3}$ The solution for $\nu=0$ was found in the conformal frame in Ref. [28]. Recently, a four dimensional black hole dressed with a conformally coupled scalar field has been obtained in [29] for a positive cosmological constant.
} 
The event horizon is located at

$$
r_{+}=B \Theta_{\nu}
$$

where the constant $\Theta_{\nu}$ is expressed in terms of $z=1+i \sqrt{\nu}$, as

$$
\Theta_{\nu}=2(z \bar{z})^{2 / 3} \frac{z^{2 / 3}-\bar{z}^{2 / 3}}{z-\bar{z}} .
$$

As a function of $\nu, \Theta_{\nu}$ is monotonically increasing, and asymptotically grows as $\sqrt{\nu}$. The causal structure of this geometry is identical to that of the non-rotating BTZ black hole [22].

The mass of this black hole can be obtained from the quasilocal stressenergy tensor (15) choosing $u^{\mu}=\frac{1}{N_{\Sigma}} \delta_{t}^{\mu}$ in the surface integral (16), which now reads

$$
\begin{aligned}
M & =M_{G}+M_{\phi}=-\lim _{r \rightarrow \infty} \int_{S^{1}} r d \varphi \sqrt{-g_{t t}} g_{t t}\left(T_{G}^{t t}+T_{\phi}^{t t}\right), \\
& =\frac{1}{8 \pi G} \lim _{r \rightarrow \infty} \int_{S^{1}} r d \varphi \sqrt{-g_{t t}}\left(\left(-\frac{1}{r \sqrt{g_{r r}}}+\frac{1}{l}\right)+\frac{1}{l}\left(\phi^{2}-\frac{2 l}{\sqrt{g_{r r}}} \phi \partial_{r} \phi\right)\right) .
\end{aligned}
$$

Note that the contribution coming from the matter piece

$$
M_{\phi}=\frac{1}{2 G l^{2}}\left(B r-B^{2}\right),
$$

has a linearly divergent term which is exactly cancelled by the one appearing in $M_{G}$, which is given by

$$
M_{G}=\frac{1}{2 G l^{2}}\left(-B r+\frac{B^{2}(7+3 \nu)}{4}\right),
$$

and therefore, the black hole mass is

$$
M=\frac{3 B^{2}}{8 G l^{2}}(1+\nu) .
$$

This result coincides with the expression (77) obtained from the Hamiltonian formalism for $\xi=\partial_{t}$, i.e., $Q\left(\partial_{t}\right)=M$. 


\subsection{New solutions}

For the range $\nu \leq 0$, an independent static circularly symmetric solution with a nontrivial scalar field exists ${ }^{4}$. The scalar field is given by

$$
\phi=\operatorname{arctanh}\left(\sqrt{\frac{B}{f(r)+B \sqrt{-\nu}}}\right)
$$

with

$$
f(r)=\frac{1}{2}\left(r-B(\sqrt{-\nu}-1)+\sqrt{(r-B(\sqrt{-\nu}-1))^{2}+4 B \sqrt{-\nu} r}\right),
$$

so that $\alpha$ in Eq. (41) differs from $-\frac{2}{3}$, the value it attains in the black hole case, but now depends on the parameter $\nu$ :

$$
\alpha=-\frac{1+3 \sqrt{-\nu}}{6} \text {. }
$$

The metric is given by

$$
d s^{2}=-\frac{r^{2}}{l^{2}} d t^{2}+\frac{l^{2}(f+B(\sqrt{-\nu}-1))^{2}(f+B \sqrt{-\nu})^{2}}{f^{2}\left(f^{2}-B(B-2 f) \sqrt{-\nu}-B^{2} \nu\right)^{2}} d r^{2}+r^{2} d \varphi^{2}
$$

with asymptotic behavior of the form (5). The integration constant $B$ is nonnegative, and for $B=0$, this solution reduces to the massless BTZ black hole with $\phi=0$. The geometric behavior of (24) radically varies, depending on the range of the parameter $\nu$.

\subsubsection{The Nullnut}

For the range $\nu<-1$, the potential looks like a "Mexican hat". In this case, the scalar field (22) is regular everywhere, and the metric (24) possesses a timelike killing vector whose norm vanishes at $r=0$. Note that, under a

\footnotetext{
${ }^{4}$ In the conformal frame [20, this solution corresponds to a massless BTZ black hole dressed with a scalar field given by $\hat{\phi}=\sqrt{\frac{B}{r+B \sqrt{-\nu}}}$, which for $\nu<0$ is regular everywhere. For the case $\nu=0$ 30, 31, the scalar field diverges at the origin.
} 
suitable time rescaling, the line element around $r=0$ can be written as a massless BTZ black hole with an effective AdS radius $\tilde{l}=l \frac{\sqrt{-\nu}-1}{\sqrt{-\nu}}$,

$$
d s_{r \rightarrow 0}^{2}=-\frac{r^{2}}{\tilde{l}^{2}} d t^{2}+\frac{\tilde{l}^{2}}{r^{2}} d r^{2}+r^{2} d \varphi^{2},
$$

This means that the geometry is smooth, as can be seen from the behavior of the Ricci scalar near the origin

$$
R_{r \rightarrow 0}=-\frac{6}{\tilde{l}^{2}}+O\left(r^{2}\right) .
$$

This geometry has been dubbed "nullnut" because it has a nut on the null curve $r=0$. Its causal structure is the same as for the massless BTZ black hole, irrespective of the value of the integration constant $B$. As can be foreseen through the invariance under boosts in the $t-\varphi$ plane, this solution, independently of the integration constant $B$, has a vanishing mass. This can be explicitly checked from the quasilocal stress-energy tensor (15) by choosing $u^{\mu}=\frac{1}{N_{\Sigma}} \delta_{t}^{\mu}$ in the surface integral (16). In this case, the contribution coming from the matter piece,

$$
M_{\phi}=\frac{B r}{2 l^{2}}+\frac{B^{2}(1-3 \sqrt{-\nu})}{4 l^{2}},
$$

is exactly cancelled by the contribution of the gravitational sector, that is, $M_{G}=-M_{\phi}$, yielding zero total mass. Owing to this fact, these configuration can be regarded as a sort of degenerate ground state.

For the range $-1 \leq \nu \leq 0$, the metric (24) describes a different geometry, because it has what we consider to be a mild naked singularity at $r=0$, since its mass vanishes, and it has a finite Euclidean action.

\section{Thermodynamics from the Euclidean ac- tion}

Since our action (8) has been regularized by the presence of the counterterm (14), in the semiclassical approximation the partition function is determined by the exponential of the Euclidean action, $Z=\exp (I)$, evaluated on the classical solution, without the need of a background substraction. 
Hence, the thermodynamics for the scalar black hole (1819) can be read from the Euclidean action evaluated on the Wick-rotated solution.

On shell, the bulk term reduces to

$$
\begin{aligned}
I_{\text {Bulk }} & =\frac{2}{\pi G} \int_{M} d^{3} x \sqrt{g} V(\phi) \\
& =-\frac{\beta}{2 G l^{2}} \lim _{r_{0} \rightarrow \infty} \int_{r_{+}}^{r_{0}} d r \frac{r\left[(H+B)^{3}+\nu B^{3}\right]}{H^{2}(H+2 B)} \\
& =-\frac{\beta}{4 G l^{2}}\left[r_{0}^{2}+2 B r_{0}-B^{2}(5+3 \nu)\right],
\end{aligned}
$$

and, when the cut-off $r_{0} \rightarrow \infty$, the surface terms read

$$
\begin{aligned}
\frac{1}{8 \pi G} \int_{\partial M} d^{2} x \sqrt{\gamma} \Theta & =\frac{\beta}{4 G l^{2}}\left[2 r_{0}^{2}+4 B r_{0}-B^{2}(7+3 \nu)\right], \\
\frac{1}{8 \pi G} \int_{\partial M} d^{2} x \frac{\sqrt{\gamma}}{l} & =\frac{\beta}{4 G l^{2}}\left[r_{0}^{2}-\frac{3}{2} B^{2}(1+\nu)\right], \\
\frac{1}{8 \pi G l} \int_{\partial M} d^{2} x \sqrt{-\gamma}\left(2 l \phi \hat{\eta}^{M} \partial_{M} \phi-\phi^{2}\right) & =\frac{\beta}{4 G l^{2}}\left[2 B^{2}-2 B r_{0}\right] .
\end{aligned}
$$

As the scalar black hole solution, described by (18) and (19), has an inverse Hawking temperature given by

$$
\beta=\frac{2 \pi l^{2}}{3 B} \frac{\Theta_{\nu}}{(1+\nu)},
$$

the Euclidean action is

$$
I=-\beta F=\frac{\pi \Theta_{\nu}}{4 G} B,
$$

and consequently, the counterterm prescription reproduces the expected thermodynamic expression for the free energy. Indeed, the mass in Eq.(21) and the entropy are recovered from (26) through

$$
\begin{aligned}
M & =-\frac{\partial I}{\partial \beta}=\frac{3 B^{2}}{8 G l^{2}}(1+\nu), \\
S & =\left(1-\beta \frac{\partial}{\partial \beta}\right) I=\frac{\pi r_{+}}{2 G}=\frac{A}{4 G} .
\end{aligned}
$$

An analogous computation shows that, for the nullnut solution given by (22) and (24) with $\nu<-1$, the Euclidean action vanishes. This is consistent with the fact that the nullnut has vanishing mass and temperature, as well as a null entropy. 


\subsection{Thermal Decay}

Note that the specific heat of the scalar black hole is given by $C=\partial M / \partial T=$ $\frac{\pi}{2} r_{+}$, which is always positive. This means that the scalar black hole can always reach thermal equilibrium with a heat bath. However, for a fixed temperature, apart from the scalar black hole (SBH), a BTZ black hole with a vanishing scalar field can also be at equilibrium with the heat bath. This raises the question of whether the scalar black hole could decay into the BTZ black hole. To examine this question, one needs to evaluate the difference between their respective free energies.

As both geometries approach AdS at infinity, the matching of the temperatures leads to the following relationship between both horizon radii:

$$
r_{+}^{S B H}=\frac{\Theta_{\nu}^{2}}{3(1+\nu)} r_{+}^{B T Z} .
$$

The action for the scalar black hole is given by (26), and the action for BTZ can be readily obtained in the same way: $I_{B T Z}=\pi r_{+}^{B T Z} / 4 G$. Therefore their difference

$$
I_{S B H}-I_{B T Z}=\frac{\pi}{4 G}\left[\Theta_{\nu}-\frac{3(1+\nu)}{\Theta_{\nu}}\right] B,
$$

which is always negative since $\Theta_{\nu}-\frac{3(1+\nu)}{\Theta_{\nu}}<0$ for the range $\nu>-1$. This means that there is a nonvanishing probability for the decay of a scalar black hole into the BTZ black hole, induced by the thermal fluctuations, irrespective of the value of the horizon radius. Furthermore, since

$$
\frac{M_{S B H}}{M_{B T Z}}=\frac{S_{S B H}}{S_{B T Z}}=\frac{\Theta_{\nu}^{2}}{3(1+\nu)}<1,
$$

in the decay process, the scalar black hole absorbs energy from the thermal bath, thus increasing its horizon radius, and consequently its entropy. This suggests that in this process, the scalar field is, in some sense, absorbed by the black hole.

Analogously, since the nullnut solution, given by (22) and (24), has vanishing temperature and action, there is probability of decaying into a BTZ black hole. Hence, in a similar way, the nullnut would be able to absorb its own scalar field. 


\subsection{Central charge}

Note that, even though the fall-off of the fields at infinity, given by (41) and (15), is slower than that of a localized distribution of matter, this set of conditions is also left invariant under the Virasoro algebra generated by (11). Moreover, although the expression for canonical charges differs form the one found in [4, it was shown in [20] that their algebra is identical, i.e., two copies of the Virasoro algebra with exactly the same central extension. This follows from the results of Ref. [32, which states that the bracket of two charges provides a realization of the asymptotic symmetry algebra with a possible central extension. For the class of potentials which are consistent with the modified asymptotic behavior (4) and (5), the massless BTZ black hole with vanishing scalar field corresponds to the ground state. Thus, the central charge can be determined by computing the variation of the charges on the vacuum. It is simple to check that the same result is obtained for the nullnut solution given in Sec. 3.2.1

The purpose of this section is to show that the central charge can also be obtained in the context of the AdS/CFT correspondence. The latter associates the Brown-York quasilocal stress-energy tensor $T^{\mu \nu}$ with the stressenergy tensor of a conformal field theory living at the spacetime boundary. For the three-dimensional cases considered here, the boundary metric is conformal to a flat cylinder, i.e., $d s^{2}=-\frac{d t^{2}}{l^{2}}+d \varphi^{2}=-d x^{+} d x^{-}$, with $x^{ \pm}=\frac{t}{l} \pm \varphi$. It is well known that under diffeomorphisms of the form

$$
\begin{aligned}
& x^{+} \rightarrow x^{+}-\xi^{+}\left(x^{+}\right), \\
& x^{-} \rightarrow x^{-}-\xi^{-}\left(x^{-}\right),
\end{aligned}
$$

the $T_{++}$component of the stress-energy tensor for a two-dimensional CFT transforms as

$$
\delta_{\xi} T_{++}=\left(2 \partial_{+} \xi^{+} T_{++}+\xi^{+} \partial_{+} T_{++}\right)-\frac{c}{24 \pi} \partial_{+}^{3} \xi^{+}
$$

where the last term is the quantum anomaly. Hence, the central charge $c$ can be obtained by identifying the Brown-York tensor in Eq. (15) with that of the dual CFT. Indeed, making the variation of (15) using the asymptotic Killing vectors given by

$$
x^{+} \rightarrow x^{+}-\xi^{+}-\frac{l^{2}}{2 r^{2}} \partial_{-}^{2} \xi^{-},
$$




$$
\begin{aligned}
x^{-} & \rightarrow x^{-}-\xi^{-}-\frac{l^{2}}{2 r^{2}} \partial_{+}^{2} \xi^{+}, \\
r & \rightarrow r+\frac{r}{2}\left(\partial_{+} \xi^{+}+\partial_{-} \xi^{-}\right),
\end{aligned}
$$

and evaluating the expression for the ground state (for which $T_{\mu \nu}$ vanishes) one obtains

$$
\delta_{\xi} T_{++}=-\frac{l}{16 \pi G} \partial_{+}^{3} \xi^{+}
$$

Comparison of (28) with (27) yields the same expression as one obtains for pure gravity with a negative cosmological constant,

$$
c=\frac{3 l}{2 G}
$$

and hence, the Brown-Henneaux central charge is recovered from the AdS/CFT correspondence. It is simple to check that the counterterms explicitly containing the scalar field do not contribute to the variation of the quasilocal stress-energy tensor, and also that the same result would hold if we had chosen the nullnut solution as the ground state.

In the case of pure gravity with the standard asymptotic conditions, the central charge was first obtained through the AdS/CFT correspondence by Henningson and Skenderis [8].

As was noted in [20, it is worth mentioning that if one naively follows the Cardy-Strominger approach [33], for the black hole solution given by (18) and (19), a value for the entropy that always exceeds the semiclassical result is obtained, thus violating in this way the holographic bound [34, 35].

\section{Discussion and comments}

A finite action for three-dimensional gravity with a minimally coupled selfinteracting scalar field has been constructed. Since the fall-off of the fields at the asymptotic region was assumed to be slower than that of a localized distribution of matter, the counterterm Lagrangian needed to render the action finite acquires contributions depending explicitly on the scalar field. This means that the quasilocal conserved charges also acquires a non trivial contribution coming from the matter sector. This fact is in agreement with results obtained via the Hamiltonian [20] as well as covariant methods [36]. 
The same central charge as for pure gravity was found by means of the AdS/CFT correspondence. The required counterterms were found by demanding that the action attain an extremum for the boundary conditions mentioned above, and this also insures the convergence of the action. A treatment of this method in a more general settings will appear elsewhere.

The asymptotic conditions considered here correspond to exact solutions including black holes. Had we assumed different asymptotic behavior for the fields, different counterterms would be found. This problem has also been explored in three and higher dimensions [37, 38, 39].

The possibility of a phase transition between black holes and solutions with naked singularities [40] in three dimensions, as well as its relevance for the AdS/CFT correspondence have been recently explored in Ref. 41]. A more general discussion of the problem of the thermodynamical properties of naked singularities in this model is currently under study.

\section{Acknowledgments}

The authors are grateful to Juan Crisóstomo, Marc Henneaux and Jorge Zanelli for helpful remarks, and also to Kostas Skenderis and Jacek Wiśniewski for pointing out to us some references. This work is partially funded by grants 1010446, 1010449, 1010450, 1020629, 7010446, 7010450 from FONDECYT. Institutional support to the Centro de Estudios Científicos (CECS) from Empresas CMPC is gratefully acknowledged. CECS is a Millennium Science Institute and is funded in part by grants from Fundación Andes and the Tinker Foundation.

\section{References}

[1] L. F. Abbott and S. Deser, Nucl. Phys. B 195, 76 (1982).

[2] A. Ashtekar and A. Magnon, Class. Quantum Grav. 1 (1984) L39

[3] M. Henneaux and C. Teitelboim, Commun. Math. Phys. 98, 391 (1985).

[4] J. D. Brown and M. Henneaux, Commun. Math. Phys. 104, 207 (1986).

[5] M. Henneaux, in Proceedings of the Fourth Marcel Grossmann Meeting on General Relativity, R. Ruffini (ed.). (Elsevier Science Publishiers B. V., 1986). 
[6] S. W. Hawking and G. T. Horowitz, Class. Quant. Grav. 13, 1487 (1996).

[7] B. Julia and S. Silva, Class. Quant. Grav. 15, 2173 (1998).

[8] M. Henningson and K. Skenderis, JHEP 9807, 023 (1998).

[9] V. Balasubramanian and P. Kraus, Commun. Math. Phys. 208, 413 (1999).

[10] R. B. Mann, Phys. Rev. D 60, 104047 (1999).

[11] R. Emparan, C. V. Johnson and R. C. Myers, Phys. Rev. D 60, 104001 (1999).

[12] R. Aros, M. Contreras, R. Olea, R. Troncoso and J. Zanelli, Phys. Rev. Lett. 84, 1647 (2000).

[13] R. Aros, M. Contreras, R. Olea, R. Troncoso and J. Zanelli, Phys. Rev. D 62, 044002 (2000).

[14] A. Ashtekar and S. Das, Class. Quant. Grav. 17, L17 (2000).

[15] G. Barnich and F. Brandt, Nucl. Phys. B 633, 3 (2002).

[16] G. Barnich, "Boundary charges in gauge theories: Using Stokes theorem in the bulk", arXiv:hep-th/0301039.

[17] J. M. Maldacena, Adv. Theor. Math. Phys. 2, 231 (1998) [Int. J. Theor. Phys. 38, 1113 (1999)].

[18] S. S. Gubser, I. R. Klebanov and A. M. Polyakov, Phys. Lett. B 428, 105 (1998).

[19] E. Witten, Adv. Theor. Math. Phys. 2, 253 (1998).

[20] M. Henneaux, C. Martínez, R. Troncoso and J. Zanelli, Phys. Rev. D 65, 104007 (2002).

[21] J. D. Brown and J. W. York, Phys. Rev. D 47, 1407 (1993).

[22] M. Bañados, C. Teitelboim and J. Zanelli, Phys. Rev. Lett. 69, 1849 (1992). 
[23] C. Martínez, C. Teitelboim and J. Zanelli, Phys. Rev. D 61, 104013 (2000).

[24] A. Ashtekar, J. Wisniewski and O. Dreyer, "Isolated horizons in 2+1 gravity", arXiv:gr-qc/0206024.

[25] P. Breitenlohner and D. Z. Freedman, Annals Phys. 144, 249 (1982).

[26] L. Mezincescu and P. K. Townsend, Annals Phys. 160, 406 (1985).

[27] T. Regge and C. Teitelboim, Annals Phys. 88, 286 (1974).

[28] C. Martínez and J. Zanelli, Phys. Rev. D 54, 3830 (1996).

[29] C. Martínez, R. Troncoso and J. Zanelli, Phys. Rev. D 67, 024008 (2003).

[30] M. Natsuume, T. Okamura and M. Sato, Phys. Rev. D 61, 104005 (2000).

[31] E. Ayón-Beato, A. García, A. Macías and J. M. Pérez-Sánchez, Phys. Lett. B 495, 164 (2000).

[32] J. D. Brown and M. Henneaux, J. Math. Phys. 27, 489 (1986).

[33] A. Strominger, JHEP 9802, 009 (1998).

[34] G. 't Hooft, "Dimensional Reduction In Quantum Gravity". Essay dedicated to Abdus Salam. Published in Salamfest 1993:0284-296. arXiv:gr-qc/9310026.

[35] L. Susskind, J. Math. Phys. 36, 6377 (1995).

[36] G. Barnich, "Conserved charges in gravitational theories: Contribution from scalar fields". To appear in the proceedings of 3rd International Sakharov Conference on Physics, Moscow, Russia, 24-29 Jun 2002. arXiv:gr-qc/0211031.

[37] S. Nojiri, S. D. Odintsov and S. Ogushi, Phys. Rev. D 62, 124002 (2000).

[38] S. de Haro, S. N. Solodukhin and K. Skenderis, Commun. Math. Phys. 217, 595 (2001).

[39] M. Berg and H. Samtleben, JHEP 0212, 070 (2002). 
[40] S. Das, J. Gegenberg and V. Husain, Phys. Rev. D 64, 065027 (2001).

[41] S. Das, J. Gegenberg and V. Husain, Int. J. Mod. Phys. D 10, 807 (2001). 